\title{
Optimization of Production Planning for a Flexible Assembly Technology on a Mechatronics Line
}

\author{
Octavian DUCA ${ }^{1,3}$, Claudiu BIDIC $\breve{A}^{3 *}$, Eugenia MINC $\breve{A}^{2,3}$, \\ Valentin GURGU' ${ }^{1}$, Marius PĂUN', Florin DRAGOMIR ${ }^{2}$ \\ ${ }^{1}$ Institute of Multidisciplinary Research for Science and Technology, "Valahia” University of Târgoviște \\ octavian.duca@valahia.ro,valentin.gurgu@valahia.ro,marius.paun@valahia.ro \\ ${ }^{2}$ Department of Automation, Computer Science and Electrical Engineering, "Valahia" University of Târgoviște \\ eugenia.minca@valahia.ro, florin.dragomir@valahia.ro \\ ${ }^{3}$ The School for Doctoral Studies in Fundamental and Engineering Sciences, \\ "Dunărea de Jos" University of Galați \\ claudiu.bidica@yahoo.com (*Corresponding author)
}

\begin{abstract}
This paper focuses on an optimal production planning algorithm solution for an integrated flexible manufacturing system (IFSM) assisted by robotic systems. The assembly system is composed of 6 individual interconnected workstations, of which two cells equipped with six degree of freedom industrial robotic manipulators (IRM). Parallel with the interconnected workstations a SCARA robotic transportation system (SRTS) permits the movement of products forward and backward between stations. The entire system permits the production of two product types on two different production processes. One production process takes place completely in an assembly cell equipped with an ABB IRM and can produce only one product type. The other production process takes place on the interconnected stations, of which one is equipped with a Fanuc IRM and it can assembly both types of products being assisted by the SRTS in the production. An IFSM interconnected server is dedicated to the centralized control of the two parallel manufacturing processes, on-line interconnected station manufacturing and flexible cell manufacturing. As a result of introducing an algorithm aimed at minimizing the total production time, an optimized production planning is obtained on the two parallel flows, based on the request taken from the customer.
\end{abstract}

Keywords: Industrial robots, Industry 4.0, IoT, Petri nets, Robotic manipulators, Information and Communication Technology.

\section{Introduction}

Currently, industrial manufacturing is characterized by various trends, the most important being the increase of customer demand for personalised products (Westkämper \& Löffler, 2016) (Sousa \& Silveira, 2019), and increasing customization to the detriment of mass production, which requires an increase in production flexibility. Thus, a strategy to meet these customer requirements is to increase the variety and different options of product personalisation (Vogel, 2017), (Esheiba et al., 2019). This leads to a decrease in the manufacturing life of products and increase pressure to develop and design new ones (Lee et al., 2012). In recent decades, small and medium-sized enterprises have increasingly started to focus on innovation to achieve their strategic goals (Ceptureanu et al., 2020). The variety of products and their rapid development require the application of innovative technologies for their implementation and production (Nagy et al., 2018). Proper use of IT can enhance potential knowledge absorptive capacity (Popescu et al., 2019). Thus, several production information and telecommunication technologies have been applied for easier management (Piccarozzi et al., 2018), and new opportunities for product customization (Brusaferri et al., 2011) (Hernandez-Martinez \& Puga-Velazquez, 2013).
Thus, increased product customization leads to a growing risk of material stocks shortages, many of the customized products requiring different materials (Tiwong et al., 2019). On the other hand, in industry the space dedicated to storage operations is usually higher as percentage than the production space (Roscoe \& Baker, 2013), being much more inactive (Luo, et al., 2019), (Mourtzis et al., 2019). Thus, it is necessary to implement concepts such as Just-in-Time (Geismar et al., 2011), which aim to reduce inventory to the lowest possible level for manufacturing (Boysen, 2010), (Obermaier \& Donhauser, 2012). Reducing stocks also leads to an increased efficiency, some simplifications of production planning and even a reduction of the production costs (Bouabid et al., 2012), (Rivera-Gómez et al., 2019).

Another way to reduce stocks is to minimise material losses by disassembling defective products (Poschmann et al., 2020), (Minca et al., 2014). Disassembly can be performed both in the assembly stations (Filipescu et al., 2020), through reversible processes (Minca, et al., 2015), (Metea et al., 2019) and separately in special disassembly stations (Duca et al., 2019), (Dragomir et al., 2019). This leads to a decrease in losses and, as a result, an increased profitability (Ren et al., 2019). 
But for a proper use of disassembly to minimise the stocks it is necessary to digitalise production (Westkämper et al., 2013) to allow rapid information monitoring and transmission during a production process (Basir et al., 2019). Thus, a recent trend in this direction is the integration and connection of industrial manufacturing systems with the "Internet of Things and Services" to obtain a high degree of flexibility and efficiency (Kagermann et al., 2013), (Castano et al., 2019). Based on these premises, the Industry 4.0 concept was developed, first by Germany as a strategic economy development project, being adopted by other states and industries under different names and forms. This strategy is considered a potential factor for a fourth industrial revolution (Kagermann et al., 2013), (Okano, 2017). Applying the concepts of "Internet of Services and Things" and "Cyber-Physical systems" to industrial manufacturing systems leads to necessary changes (Cogliati et al., 2018) and improvements following the concepts of Industry 4.0 (Satoglu et al., 2017), (Raposo et al., 2018). But the application of these concepts integrates special features to the manufacturing systems, which leads to new requirements and challenges in the modelling and analysis of this systems (Zezulka et al., 2018), (Telukdarie et al., 2018). In order to achieve optimal results, the modelling methods must consider the special characteristics of the manufacturing systems with Industry 4.0 concepts implemented (Herceg et al., 2020), (Gharge et al., 2020).

Most flexible manufacturing applications use metaheuristic or heuristic algorithms for production planning and optimization (Abidi et al., 2020), (Zhou et al., 2020). The use of heuristic and metaheuristic algorithms detrimental to mathematical models is determined by the need to define constraints for the implementation of the mathematical model. Putting planning problems in terms of constraints and variables, several papers, focus on its expression in mathematical models. In (Rezig et al., 2020) an optimal planning model is proposed in the conditions of production and maintenance constraints detailing a new form of approach that maps a simulation. In the case of manufacturing flow, storage problems and budders in (Trabelsi et al., 2011) a mathematical model with different types of black constraints is proposed. Also, since in manufacturing flexibility is needed in process plans, in (Özgüven et al., 2010) a mathematical approach is proposed.
This article presents an optimized manufacturing planning for a flexible and reversible assembly/ disassembly process on a laboratory mechatronics line (ML), in accordance with Industry 4.0 concepts. The flexibility of the production line is given by two factors. First of all, it is possible to produce multiple types of customized products based on customer request. The second is the possibility to produce different types of products using dedicated equipment or the same equipment based on the work volume. For using the same equipment for multiple production, a special transportation system is used. This way, the product is transported on a previous station for a new series of operation.

The rest of the paper is organized as follows. The structure of the production line and the production process is presented in Section 2. Section 3 presents a Petri network (PN) modelling of the manufacturing line, in Section 4 an optimization model of the production planning is explained and Section 5 offers the conclusion and presents the implementation of Industry 4.0 concept in the production process through various ML subsystems.

\section{Production Planning for Generalized Flexible Assembly System}

In this paper a scheduling production framework is proposed for an educational IFMS, based on the minimization of the manufacturing time for a known volume of two product types, Type 1 and Type 2. In the proposed model, before the starting of the production, a request with the volume of Type 1 and Type 2 products is received directly from the client, by a local database. The generalized manufacturing system composed of assembly workstations, with two parallel production processes: a flow flexible manufacturing (FFM) composed from interconnected assembly workstations, and a flexible manufacturing cell (FMC). It is considered that Type 1 can be assembled either on FMC or on FFM while Type 2 only on FFM. Based on the manufacturing times of the two products and the required production volume a production planning is proposed for obtaining a minimal production time.

The manufacturing of Type 1 product on the FFM is obtained by moving back the product 
over a known number of stations, for a new set of operations. both product types assembled on FMC or FFM has to pass through the Quality Test, located just before the final warehouse.

This determines the volume of Type 1 products which involves the introduction of waiting times in the production of FFM. These delays are introduced at the return points of Type 1 products coming from FFM and FMC, and introduced before the Quality Test. Based on the synchronization between the assembly of Type 1 products on FFM and FMC, for obtaining the minimal production time a production planning algorithm is proposed.

\subsection{Assumptions}

In the case of the proposed IFMS assumptions were made to simplify or clarify constrains imposed in the operation of the line:

A1. The ML is a deterministic line, determined by the nature of operation times (times known with certainty);

A2. The volume of Type 1 and Type 2 products to be assembled is initially known;

A3. The influence of customization of Type 1 and Type 2 products over the production time is negligible;

A4. The IFMS works in two parallel manufacturing flows, FMC and FFM, that meet at a Quality Test point;

A5. The FMC and FFM production flows start simultaneously;

A6. The product from FMC has priority in being processed by the Quality Test, in comparation with the FFM product;

A7. Only Type 1 product will be assembled on the FCM, while both Type 1 and Type 2 products will be assembled on the FFM;

A8. The FMC manufacturing flow contains only handling / assembly/ transport operations and does not contain delays or interruptions;

A9. From the configuration of Type 1 product on FFM, a node station is identified, belonging to FFM, from which the product is taken / transported / repositioned in a previous station;

A10.The number of workstations over which the Type 1 product is handled / transported back to be reintroduced into the FFM production flow is constant for each Type 1 product and dependent to the number of layers that are repeated in its structure;

A11. For the realization on FFM of two types of products, delays in the current flow are introduced, necessary for the reintroduction in the manufacturing flow of Type 1 products;

A12.In FFM, in the node workstations where Type 1 products are reintroduced into the production flow, the Type 1 product has priority at entering in the workstation.

\subsection{Workstation General Model}

Let's consider a general model of a workstation $k$ (Figure 1) as part of a flexible assembly line with $k \in[1, N]$ stations, $N$ representing the number of workstations in the assembly line. Let's consider a product, $\mathrm{p} \in[1, \mathrm{P}]$ assembled on a workstation, with $P$ being the production volume. Let $\mathrm{T}_{\text {trans }} \mathrm{In}_{\mathrm{k}}$ be the duration of the transport into the assembly area, and $\mathrm{T}_{\text {transOut }_{\mathrm{k}}}$ the duration of transport out of the assembly area, in a station k (Figure 1). Let $\mathrm{T}_{\text {assembly }_{\mathrm{k}}}$ be the assembly duration in station $\mathrm{k}$. In the transport in area of a workstation $\mathrm{k}$ a product can come as an in-flux input from the previous station or as a return input from one of the next stations, when the product comes back for a new assembly operation in a workstation. The same way a product leaving the station $k$ can go to the next station as an in-flux output or go back for new operations to a previous workstation as a return output.

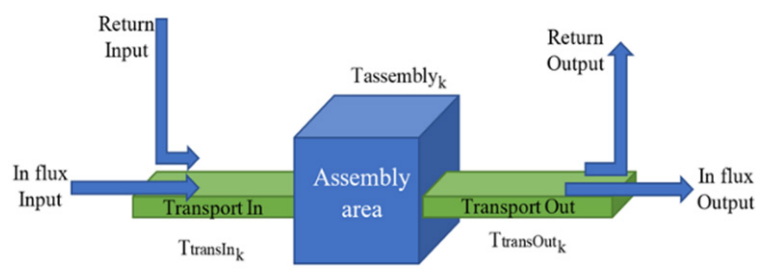

Figure 1. Workstation generalized model

For a workstation let the production time $\mathrm{T}_{\text {prod }_{\mathrm{k}}}$ be

$T_{\text {prod }_{k}}=T_{\text {transIn }_{k}}+T_{\text {wait }_{k}}+T_{\text {assembly }_{k}}+T_{\text {transOut }_{k}}$

where

$T_{\text {wait }_{k}}=\underset{j=1, N}{\max }\left(T_{\text {transIn }_{j}}+T_{\text {assembly }_{j}}+T_{\text {transOut }_{j}}\right)$

$-\left(T_{\text {transIn }_{k}}+T_{\text {assembly }_{k}}+T_{\text {transOut }_{k}}\right)$

represents the waiting time in the station given by the maximum production time on the ML. This waiting time determines the assembly time on all station to be equal. 
Let $\mathrm{T}_{\text {prod max }}$ be the maximum production time on a station:

$$
T_{\text {prod max }}=\underset{k=1, N}{\max }\left(T_{\text {transII }_{k}}+T_{\text {assembly }_{k}}+T_{\text {transOut }_{k}}\right)
$$

From (1) it is obtained

$T_{\text {prod }_{k}}=T_{\text {prod } \max }$

Based on the manufacturing time in a workstation and the assembly process, let $T_{c y c l e}^{N}$, the cycle time of a product on the FFM, be defined as

$T_{\text {cycle } e_{p}^{N}}=\sum_{k=1}^{N} \sigma_{k, p} T_{\text {prod }_{k}}+\gamma_{p} T_{\text {transport }}+\sum_{k=1}^{N} T_{\text {stop }_{k, p}}$

$\sigma_{k, p}$ the number of passes of product $p$ through a workstation $k$;

$\gamma_{p}$ the number of returns of product $p$ to a workstation, and can be defined as $\gamma_{p}=\max \left(\sigma_{k, p}\right)-1$;

$T_{\text {transport }}$ the duration of a return of product $p$;

$T_{\text {stop }_{k, p}}$ the waiting duration of product $p$ in workstation $k$ given by the introduction of a product in workstation $k+1$, defined as

$T_{\text {stop }}=\theta_{k+1, p} \cdot T_{\operatorname{prod}_{k+1}}$

where $\theta_{k+1, p}$ is the number of products that are introduced in workstation $k+1$ during the period when the product $p$ is located in station $k$.

\subsection{Assembly General Model}

The mathematical model is based on a flexible assembly line model (Figure 2). In the model it is considered a product $m, m \in[0, P]$ that needs to be transported from a station $i+i_{B a c k}$ back to workstation $i$ on FFM production flow, $i \in[1, N]$, with $i_{\text {Back }}$ being the number of workstations over which SRTS transports the product back to resume assembly. In this paper product $m$ is represented Type 1 products to be assembled on the FFM. Product $m$ will introduce two waiting time types, one that affects only the workstation usability, and one that affects also the products coming from previous stations.

Let's consider $T_{\text {halt }_{i_{i+B_{B a c k}+1, m}}}$ be the first type of waiting time introduced in a station $i+i_{\text {Back }}+1$ (Figure 2), representing the time the station waits for the next product:

$T_{\text {halt }_{i+i_{\text {Back }}+1, m}}=\delta_{i+i_{\text {Back }}+1, m} T_{\text {prod }_{i+i_{\text {Back }}}}$

where $\delta_{i+i_{\text {Back }}+1, m}$ represents the number of products that leave the manufacturing flux from workstation $i+i_{\text {Back }}$. This waiting time is a result of product $m$ leaving workstation $i+i_{B a c k}$, and does not influence the product $m+1$.

In station $i-1$ another waiting time is considered, $T_{\text {stop }_{i-1, m}}$, which is determined by the introduction of product $m$ in station $i$ (Figure 2), where:

$T_{\text {stop }}=\theta_{i, 1, m} \cdot T_{\text {prod }_{i}}$

The FMC workstation is a particular case of a typical workstation presented in subsection 3.1. As a particularity of this case, let's consider $\mathrm{T}_{\text {prodcell }}$ the manufacturing time in the FMC. For simplification it is considered a multiple of the maximum manufacturing time on the FFM:

$$
\mathrm{T}_{\text {prodcell }}=\beta \mathrm{T}_{\text {prodmax }}, \beta \in \mathrm{N}, \beta \geq 1
$$

On the FMC let's consider the assembly of a product $r$, with $r \in[0, P]$. This product is

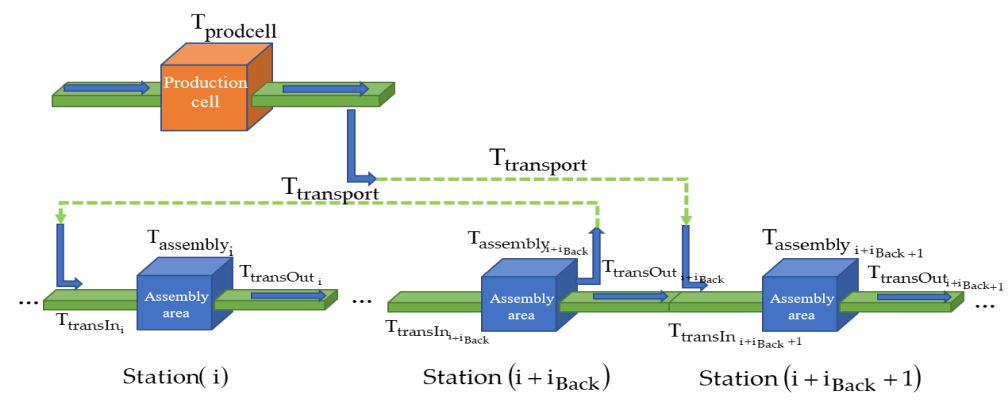

Figure 2. Flexible production model 
transported to workstation $N$ for quality control and storage. Introducing the product $r$ in station $N$, introduces a waiting time, $T_{\text {stop }_{N-1, m}}$, in station $N-1$, with

$$
\mathrm{T}_{\text {stop }_{\mathrm{N}-1, \mathrm{~m}}}=\mathrm{T}_{\text {prod, } \mathrm{N}}
$$

which repeats cyclically with $T_{\text {prodcell }}$ for $T_{\text {transport }}<T_{\text {prodcell }}$. As the product can't be placed in the ML before the completion of the production on the station, $T_{\text {transport }}$ is consider for simplification:

$T_{\text {transport }}=\lambda \cdot T_{\text {prod } \max }, \lambda \in N, \quad \lambda \geq 1$

for any transportation between FMC and FFM or on the FFM, with $\lambda$ as transport coefficient. The total manufacturing cycle time on FMC of a $r$ product can be defined as:

$T_{\text {Cyclecell }}=T_{\text {prodcell }}+T_{\text {prod }_{k}}+T_{\text {transport }}$

resulting

$$
T_{\text {Cyclecell }}=(\beta+1+\lambda) T_{\text {prod } \max }
$$

\subsection{Scheduling Model}

To obtain the best manufacturing results an optimisation model for the products repartition on the manufacturing flows is proposed. The optimization model relies on the minimization of the waiting time on both the FFM and FMC production flows, given by the of Type $1\left(T_{1}\right)$ and Type $2\left(T_{2}\right)$ products.

In equation (5) it is considered:

$\sigma_{k, p}=\sigma_{k, T_{1}}$, where $\sigma_{k, T_{1}}$ is the number of passes through a workstation $k$ of FFM for assembly operations on a Type 1 product. It is particularised: $\sigma_{k, T_{1}}=1$ for $k=\overline{1, i} \cap \overline{i+i_{B a c k}, N}$ and $\sigma_{k, T_{1}}=2$ for $k=\overline{i, i+i_{\text {Back }}}$;

$\gamma_{p}=\gamma_{T_{1}}$, where $\gamma_{T_{1}}$ is the number of returns of Type 1 product on the FFM for repeating assembly operations. In this paper it is considered that $\gamma_{T_{1}}=1$;

$T_{\text {stopk }, T_{1}}=\theta_{k+1, T_{1}} T_{p r o d_{k+1}}$ for $p=T_{1}$ in (6);

$T_{\text {transport }}=T_{\text {prod } \max }$ for $\lambda=1$ in (11).
Based on equations (5) and (6), the cycle time for Type 1 production, $\mathrm{T}_{\text {cycle }} \mathrm{T}_{1}$, on the FFM is defined as:

$$
\begin{aligned}
T_{\text {cycle }}= & \sum_{k=1}^{i+i_{\text {Back }}} T_{\text {prod }_{k}}+T_{\text {transport }} \\
& +\sum_{k=i}^{N} T_{\text {prod }_{k}}+\sum_{k=1}^{N} k+1, T_{1} \quad \operatorname{prod}_{k+1}
\end{aligned}
$$

and based on equations (4) and (14) it is obtained:

$$
\begin{aligned}
& T_{\text {cycle }_{T_{I}}}=\left(N+i_{\text {Back }}\right) T_{\text {prodmax }} \\
& \\
& \quad T+T_{\text {prodmax }}+\sum_{k=1}^{N} k+1, T_{I} \operatorname{prod}_{k+1}
\end{aligned}
$$

Similarly, for a Type 2 product, for the parameters $\sigma_{\mathrm{k}, \mathrm{T}_{2}}=1, \mathrm{k}=\overline{1, \mathrm{~N}}$ and $\gamma_{\mathrm{T}_{2}}=0$ it is obtained:

$T_{\text {cycle } T_{2}}=\sum_{k=1}^{N} T_{\text {prod }_{k}}+\sum_{k=1}^{N}, k+1, T_{2} T_{\text {prod }_{k+1}}$

resulting

$$
\mathrm{T}_{\text {cycle }}^{\mathrm{N}} \underset{\mathrm{T}_{2}}{\mathrm{~N}}=\mathrm{NT}_{\text {prod max }}+\sum_{\mathrm{k}=1}^{\mathrm{N}} \theta_{\mathrm{k}+1, \mathrm{~T}_{2}} \mathrm{~T}_{\text {prod }_{\mathrm{k}+1}}
$$

Let's consider $v_{1}$ and $v_{2}$ the volumes of Type 1 and Type 2 products, received from customer.

Let's consider $\eta$ the number of Type 1 products that will be manufactured on the FFM.

Let's consider $\mathrm{T}_{\text {TotalWait }}$ be the total waiting time introduced by $v_{1}$ products to the production volume of Type 1 and Type 2 products on the FFM flow. It can be expressed as:

$$
\begin{aligned}
T_{\text {TotalWait }}= & \sum_{T_{2}=l}^{v_{2}} \sum_{k=1}^{N} \theta_{k+1, T_{2}} \cdot T_{\text {prod }_{k+1}} \\
& +\sum_{T_{1}=1}^{\eta} \sum_{k=1}^{N} \theta_{k+1, T_{1}} \cdot T_{\text {prod }_{k+1}}
\end{aligned}
$$

To obtain a minimal manufacturing time on the FFM the total waiting time must be minimal. The total waiting time without production optimisation is

$$
T_{\text {TotalWait }}=\left.v_{1} \cdot T_{\text {prod max }}\right|_{\text {unoptimized }}
$$

Waiting time defined by the Type 1 production volume as each product introduces a waiting time equal with the manufacturing duration of the station in which the Type 1 product is introduced.

Based on equation (6), it can be observed that, if Type 1 products from the FMC are introduced on the FFM when the $\eta$ Type 1 products from 
the FFM are returned for a new set of assembly operations, these products will not induce waiting times on the FFM. Based on this observation the total waiting time can be rewritten as:

$$
T_{\text {TotalWait }}=\left.\left(v_{1}-\eta\right) T_{\text {prod max }}\right|_{\text {optimized }}
$$

Let's consider $\mathrm{T}_{\text {prodFFM }}$, the production of the $v_{2}$ Type 2 and $\eta$ Type 1 volumes on FFM. In each station, at a certain moment, there is a product in a partial production state, $\mathrm{T}_{\text {prodFFM }}$, which can be defined as:

$T_{\text {prodFFM }}=N T_{\text {prodmax }}+\frac{1}{N} \sum_{T_{2}=1}^{v_{2}-1} N T_{\text {prodmax }}$

$+\sum_{T_{2}=1}^{v_{2}} \sum_{k=1}^{N} \theta_{k+1, T_{2}} T_{\text {prod }_{k+1}}+\sum_{T_{1}=1}^{\eta} \sum_{k=1}^{N} \theta_{k+1, T_{1}} T_{\text {prod }_{k+} .}$

$+\frac{\sum_{T_{1}=1}^{\eta}\left(N+i_{\text {Back }}\right) T_{\text {prodmax }}}{N+i_{\text {Back }}}+\sum_{T_{1}=1}^{\eta} N T_{\text {prodmax }}$

Considering the assumption $\mathrm{A} 8$, as the manufacturing duration on FMC does not include waiting times and the transport and assembly operations have a known duration, to obtain an optimal manufacturing time the total waiting time needs to be minimized while equalizing the total manufacturing time on FMC and FFM. Based on the minimisation of the waiting time, the minimisation function can be defined as:

$J_{I F M S}=\min \left(T_{\text {TotalWait }}\right)$

Based on equation (22) for the equalisation of the FFM and FMC parallel flows, it must be ensured that the manufacturing times on the two flows are almost equal:

$\sum_{r=1}^{v_{l}-\eta} T_{\text {CycleCell }}-T_{\text {prodFFM }}=0$

Based on relations (12), (20) and (22) it is obtained: $\sum_{r=1}^{v_{1}-\eta}(\beta+2) T_{\text {prodmax }}-\left[2 \eta T_{\text {prodmax }}+\left(v_{2}-1\right) T_{\text {prodmax }}\right.$

$+N T_{\text {prodmax }}+\sum_{T_{2}=1}^{v_{2}} \sum_{k=1}^{N} \theta_{k+1, T_{2}} T_{\operatorname{prod}_{k+1}}$

$\left.+\sum_{T_{1}=1}^{\eta} \sum_{k=1}^{N} \theta_{k+1, T_{1}} T_{p r o d_{k+1}}\right]=0$

and based on equation (19), one obtains:

$\left(v_{1}-\eta\right)(\beta+2) T_{\text {prodmax }}-\left[\eta T_{\text {prodmax }}+v_{2} T_{\text {prodmax }}\right.$

$\left.-T_{\text {prodmax }}+N T_{\text {prodmax }}+v_{I} T_{\text {prodmax }}\right]=0$
After reducing the terms, equation (24) becomes:

$v_{1}(\beta+2) T_{\text {prod max }}-\eta(\beta+3) T_{\text {prod max }}$

$-v_{2} T_{\text {prod } \max }-N T_{\text {prod } \max }+T_{\text {prod } \max }=0$

resulting

$\eta=\frac{v_{1}(\beta+1)-v_{2}-N+1}{\beta+3}$

Starting from relation (17) let $T_{\text {sync }}$ be a synchronization period between Type 1 manufacturing on the FMC and Type 1 manufacturing on the FFM, with $c$ as synchronization factor $T_{\text {sync }}=c T_{\text {prod } \max }$.

This synchronization period illustrates the amount of time needed by Type 1 products on the FMC and FFM to arrive simultaneously in station $i+i_{\text {Back }}$. This can also be written as:

$T_{\text {sync }}=\left(T_{\text {prodcell }}+T_{\text {transport }}\right)-T_{\text {cycleT } 1} \stackrel{i+i_{\text {Back }}}{ }$

Based on equations (4) and (28) it is obtained:

$c=\beta+1-\left(i+i_{B a c k}+\theta_{i, T l}\right)$

Three major cases can be distinguished:

$c<0$, the manufacturing of Type 1 on FFM starts with $|c|$ steps before the manufacturing starts in FMC;

$c=0$, the manufacturing in FFM and FMC start simultaneously;

$c>0$, the manufacturing of Type 1 on FFM starts with $c$ steps after the manufacturing starts in FMC.

In order to use ISFM at maximum production capacity, two case templates are proposed. These templates correspond to the repetitive assembly sequences determined from the production planning optimization algorithm. Each repetitive sequence contains successive sets of data composed of: number of parts, typology and manufacture flow to be launched on (FFM or FMC):

for $\mathrm{c}<0$

1 Type 1 product on FFM

c Type2 product on FFM

1 Type 1 product on FFC

$\beta-2-\mathrm{c}$ Type2 product on FFM 
for $c \geq 0$

$1 \quad$ Type 1 product on FMC

c Type2 product on FFM

1 Type 1 product on FFM

$\beta-2$ - c Type2 product on FFM

\section{Mechatronics System for Flexible Manufacturing}

\subsection{Hardware Structure of the Mechatronics Line}

The hardware system is represented by an extended laboratory mechatronics assembly line SMART ASTI. The line is composed of 6 interconnected workstations (Figure 3a) for flexible manufacturing, equipped with two 6-DOF industrial robotic manipulators (IRM), and a SCARA robotic transportation system (SRTS) (Figure 3b). The mechatronics system is designed for assembly operations through flexible manufacturing and component recovery by disassembling. Thus, flexible assembly is performed by both flow assembly operations in certain mechatronics line (ML) workstations and complete assembly operations performed in a flexible manufacturing cell (FMC) in Station 3 (Figure 3).

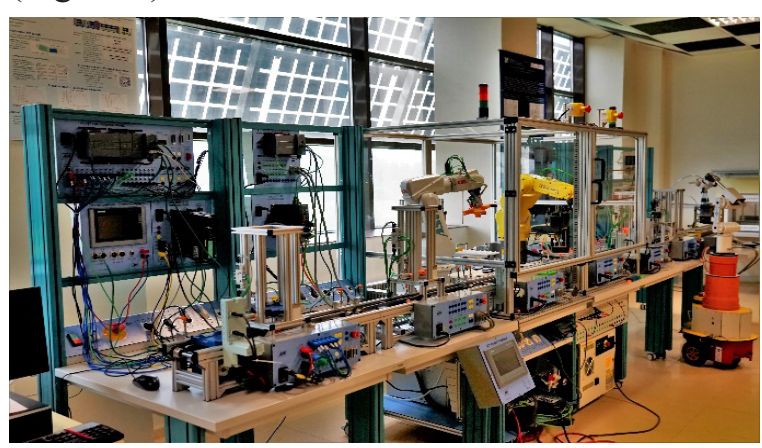

a)

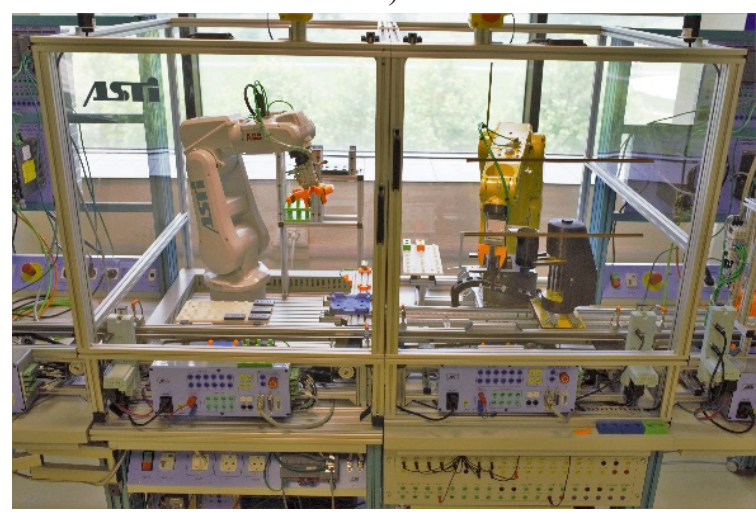

b)

Figure 3. a) IFSM equipped with IRMs; b) ABB robot (left) Fanuc robot and SRTS (right)
Stations 1, 2, 4, 5, 6 are functionally connected in the order of necessary operations for a successive assembly of components (Figure 4), a complete assembly cycle of a product being performed. To adapt in-line classic flow manufacturing technology to flow flexible manufacturing (FFM), the manufacturing system was equipped with a SRTS designed to serve the production flow through repositioning, transport and handling operations. The SRTS performs operations of repositioning and handling of a product in a previous station, for repeating assembly operations, based on the configuration of the demanded product.

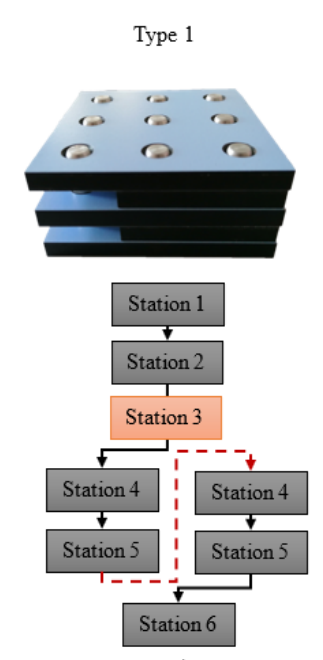

a)

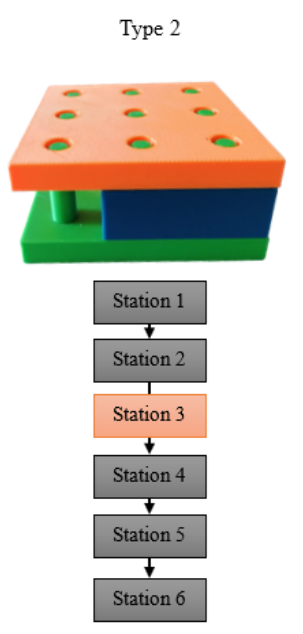

b)
Figure 4. FFM tasks on the IFMS for a) Type 1, b) Type 2 products considered in this paper

In FMC from Station 3, by equipping it with an ABB IRM and its own component warehouses, a complete assembly cycle can be executed. Considering the potential for diversification of product typologies, the design and planning of manufacturing in FMC will allow the production of complex configurations, which can be different or similar with the ones assembled FFM.

The mechatronics system connects both FFM and FMC streams to a single Quality Control (QT) point in station 6 . The two systems composing the FFM and FMC flows define an integrated flexible manufacturing system (IFMS). The flexible manufacturing is executed on both systems independently and parallel, with mutual conditioning at quality control process and starting of the production process, the intersection of the two production flows. The production diversity is assured through the control technologies present in the FFM and FMC, that communicates with the 
manipulation and transport control system of the SRTS, synchronising the systems.

The SRTS is used to transport products by bypassing some workstations or to transport the product backwards on the production flow. This allows the manufacturing of multiple configurations using a minimal number of stations, through the repetition of some assembly operation.

The IFMS is also connected to a server into the local Ethernet network (Figure 5). This server connection is used in the testing and simulation of research ideas and applications used in the implementation of Industry 4.0 concepts. Production data from the customer are processed on server applications. The information is retrieved from the customer through a client application. On the local server is also implemented a scheduling program that mased on the information from client and process information sends a resulted production schedule to the fabrication system.

\subsection{Production Process}

The principal attribute of the manufacturing on the mechatronics system is that of flexibility. The presented system can assembly multiple configurations of stratified products (Figure 4).

Are proposed two types of assembled products: with multiple internal layers (Type 1) and with a single internal layer (Type 2). The internal pieces of a product are placed based on the customer requirement. From the Type 1 product multiple product configurations can be made based on the product layers. The three internal layers are composed from two internal pieces layers and a Top part layer.

In this paper the manufacturing process of two products with custom configurations will be considered (Figure 4): multi-layers product (Type 1) and the single-layer product (Type 2). Type 1 product can be assembled on both FMC and FFM while Type 2 product can be assembled only on FFM.

The manufacturing process is presented in Figure 6 as a task diagram. The assembly tasks for the two production processes starts based on the results of the planning optimization algorithm. In Figure 6.a it can be observed the production flow of Type 2 through the workstations. After the assembly is completed, the product arrives in a quality control area where it is verified for defects using a visual analysis automatic process. The parts that pass the test are stored in Station 6 and the parts that fails it are sent for disassembling.

For Type 1 product the assembly process is different. The complete assembly is done in Station 3. To avoid the interference with Type 2 assembly process, Type 1 products are transported to the Quality Control point by the SRTS. Permits the assembly processes on the other workstations to continue uninterrupted. If the product passes the quality test it is stored with Type 2 products in Station 6. If it fails the test, the product is transported back in Station 3 by the SRTS. The Station 3 is a complete assembly/disassembly production cell that can work independently or as a part of the production line.

In the proposed manufacturing system, the flexibility is given by the possibility of assembling of two different products with distinct assembly orders without the need of hardware reconfiguration. To compensate for the hardware reconfiguration the SRTS transports the partially assembled product between some workstations based on the assembly order of the product (Figure 6.b). The operation of the SRTS is controlled locally based on the number and order

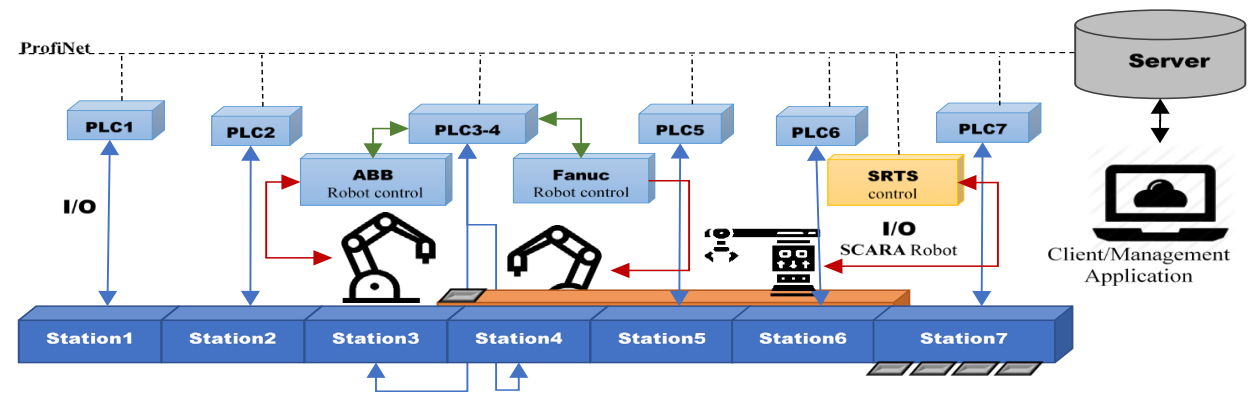

Figure 5. Stations placement and communication between them

https://www.sic.ici.ro 


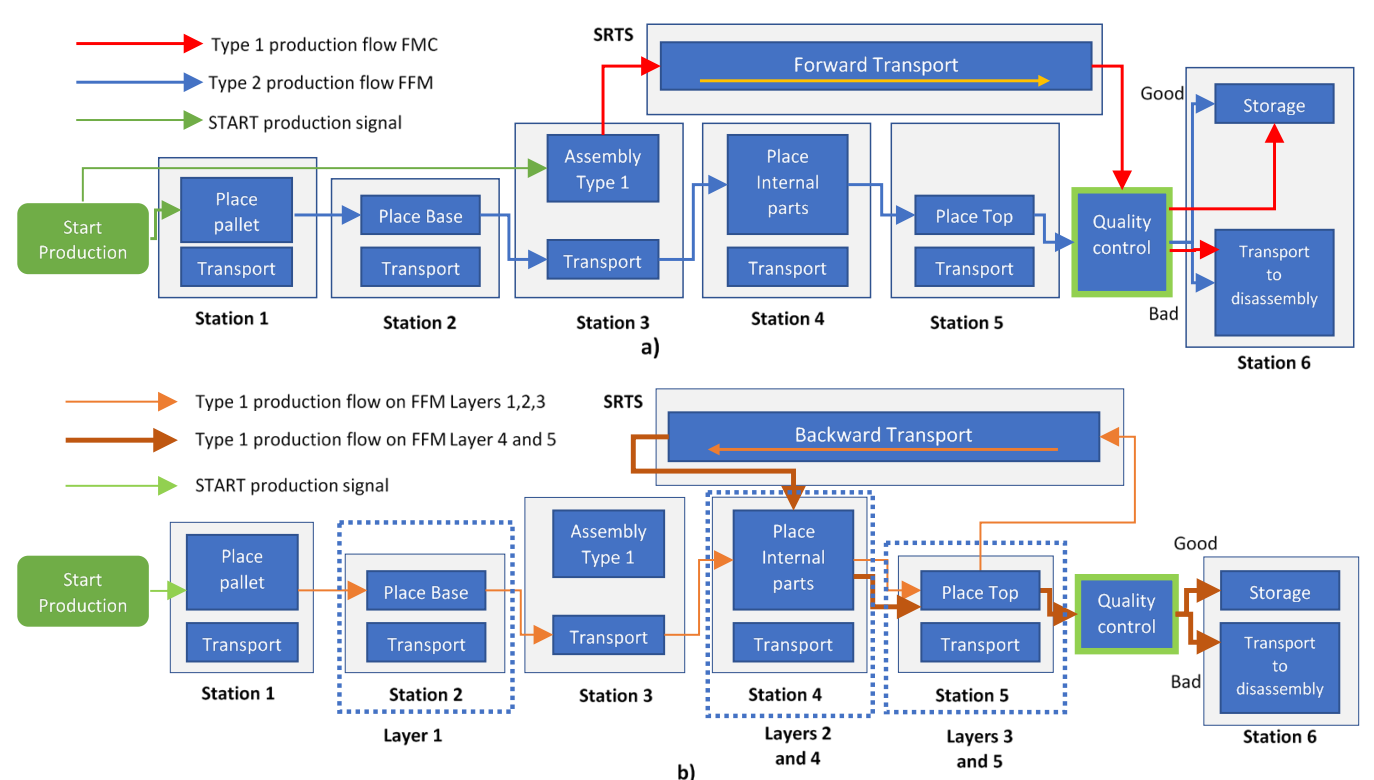

Figure 6. Task Diagram: a) Type 1 production flow on FMC and Type 2 production flow on FFM; b) Type 1 production on FFM

of the Type 1 products assembled on the FFM from the production tasks transmitted from the server. The production tasks are represented by the number, order and configuration of Type 1 and Type 2 products assembled on FMC and FFM and the order of their assembly.

\section{Case Study. Task Planning for IFMS_SMART ASTI}

For the production system presented a production of 10 Type 1 and 15 Type 2 products will be considered.

On the previous implemented production planning the distribution of the products on FMC and FFM manufacturing flows is: Type 2 products on FFM, half of Type 1 products on FFM and half of Type 1 products on FMC. The Type 1 products on FFM will be manufactured first, in parallel with Type 1 products on FMC, followed by the manufacturing of Type 2 products on FFM. For the proposed situation, for the previous production planning, a distribution of 5 Type 1 products on FMC, 5 Type 1 products on FFM and 15 Type 2 products on FFM was obtained. The manufacturing results can be observed in Figure 9a. Based on equations (19) and (20) the waiting time for the previous implemented production planning is $8 \cdot T_{\text {prod } \max }$.

For the implementation of the task planning mathematical model some of the parameters are determined from the constructive model of the product (Figure 7). From the constructive model of the ISFM, product model and the experimental results can be defined:

- the number of FFM stations $N=6$;

- $\quad$ the return station $i+i_{\text {Back }}=5$;

- $\quad$ the station to be returned to $i=4$;

- the products introduced on the production time of Type $1 \quad \theta_{i, T_{1}}=1$ after initialization and $\theta_{i, T_{I}}=O$ on initialization period;

- the production duration on FMC $i=4$.

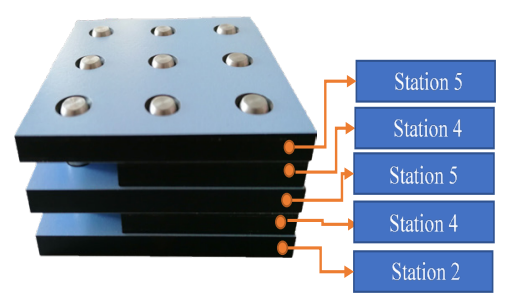

Figure 7. Type 1 product operation stations by layers

Based on equation (26) of the model, Type 1 products, which will be manufactured on the FFM, are represented by $\eta=5$ and the remaining products represented by $v_{1}-\eta=5$ will be manufactured on the FMC. 
Based on the fact that the first Type 1 product manufactured on the FMC is not delayed by any product in the initialization stage $\theta_{i, T_{l}}=0$, a factor $c=1$ is resulting, meaning that the starting of Type 1 product on the FFM is done at one stage after the start of Type 1 production on FMC. The determining of production of one Type 1 product on FMC, one Type 2 product on FFM, one Type 1 product on FFM and two Type 2 products on FFM, can be seen in Figure 8. With the introduction of a product in Station 4 the parameters change and an additional Type 2 product is introduced, in order to compensate for a new production series. In this case the first six products represent an initialization stage (Figure 8).

In the main production stage, the parameter obtains a synchronization coefficient which determines the starting of production of Type 1 both on FMC and FFM on the same time. Thus, a production series results: one Type 1 product on
FMC, one Type 1 product on FFM and three Type 2 products on FFM.

In the main production stage, the parameter $\theta_{i, T_{1}}=1$ obtains a synchronization coefficient of $c=0$ which determines a starting of production of Type 1 both on FMC and FFM on the same time. Thus, a production series results: one Type 1 product on FMC, one Type 1 product on FFM and three Type 2 products on FFM.

In Figure 9 it can be observed the production flow for the obtained results compared with those of a non-optimized situation. It can be noticed the continuous flow at the Station 6 based on the production of the FFM and FMC. It can also be seen that the waiting times induced on the production flow are kept in stations below Station 4 , obtaining a minimum waiting time. A waiting time reduction of $50 \%$ is obtained, from $8 \cdot T_{\text {prod max }}$ to $4 \cdot T_{\text {prod } \max }$. At the same time, an increase in workstation usage is obtained as the

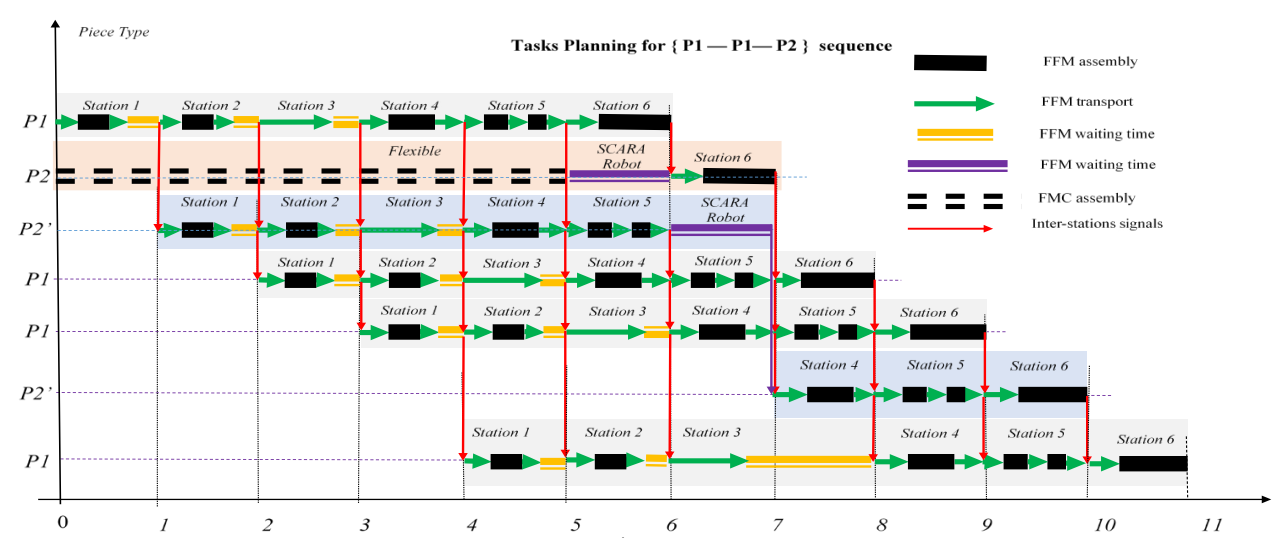

a)

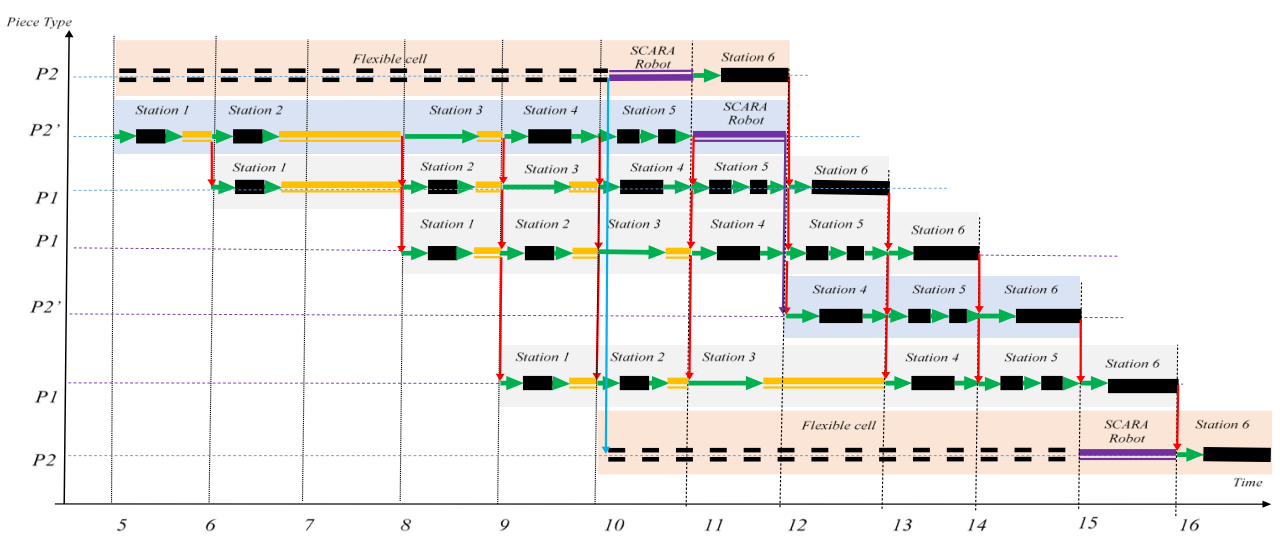

a)

Figure 8. Tasks planning on production stages: a) Initialization, b) Production cycle for repetitive sequences 


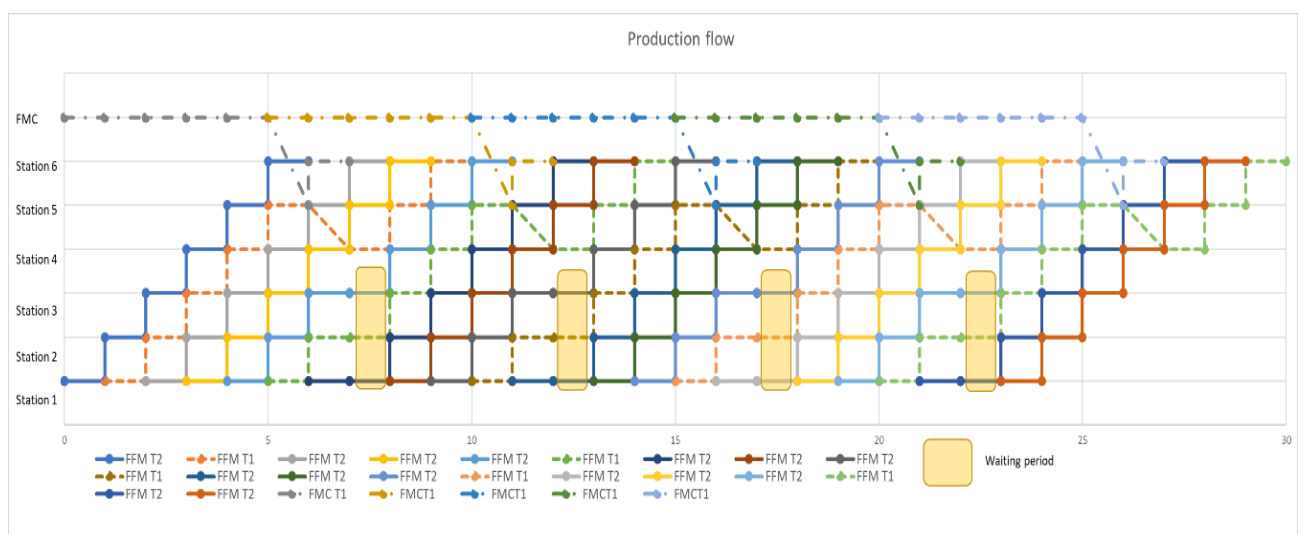

a)

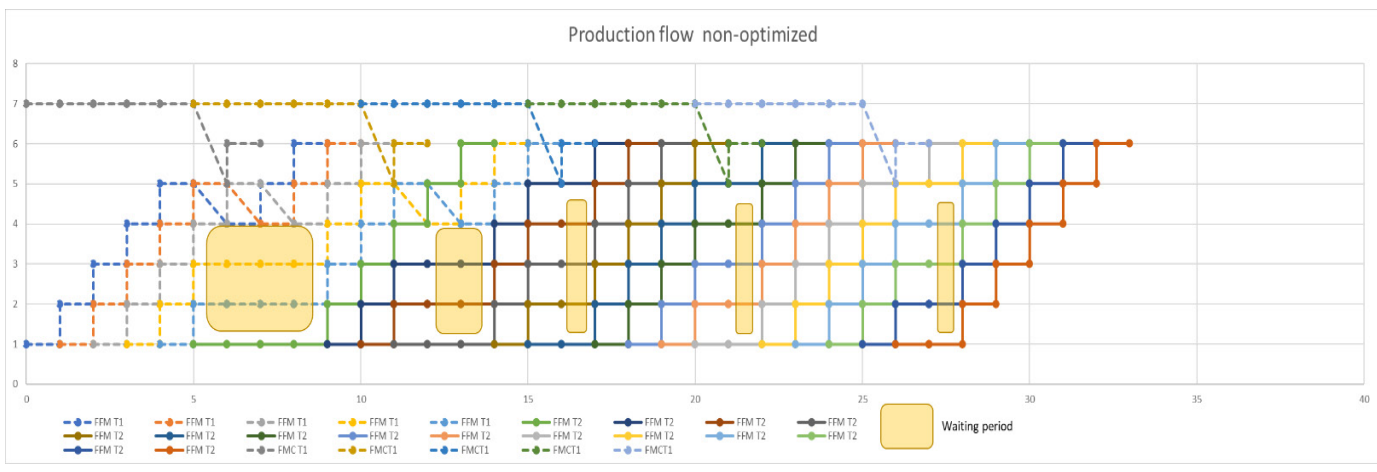

b)

Figure 9. Production Flow: optimized (a) and non-optimized (b)

waiting time is reduced to only the first three stations, as it can be seen in Figure 9. On the non-optimized situation, the two production types are done one after the other with Type 1 production on FFM first followed by Type 2 production. It can be seen that the differences in waiting times are much higher in the nonoptimized situation.

As a result of the waiting time reduction, the total production duration was reduced from a duration of $33 \cdot T_{\text {prod max }}$ to a duration of $30 \cdot T_{\text {prod max }}$ resulting to a reduction of a $9 \%$ compared with the previous implemented production planning.

\section{Conclusion}

Today, when customer demands have become extremely dynamic, production systems must be automatically adapted to the most diverse demands, through high-performance processing systems and the adaptation of manufacturing control to high productivity requirements.
The paper proposes a generalized algorithm for optimized production planning, which can be customized on production systems for flexible parallel manufacturing. The proposed algorithm was designed on an integrated system for flexible manufacturing, in which the two production systems (a flexible cell and a production system with interconnected stations) work simultaneously for a single production load/demand, relative to two product typologies. Through the optimized production planning algorithm, proposed and implemented on the flexible manufacturing system in a laboratory, the demand was decomposed into production tasks. Thus, work volumes are distributed on the two production systems, with a view to minimizing the total production time. The proposed approach has a high degree of generality, the reasoning can be extended to the manufacture of more than two types of products in the shortest time.

This article brings a scientific plus by transforming and adapting a flow manufacturing system into one 
dedicated to flexible manufacturing. Thus, with a minimum of hardware resources, a diversified and high-performance production is obtained. Moreover, by integrating in the manufacturing control the algorithm for optimizing the production planning, a flexible system is obtained that automatically adapts to a diverse production, in conditions of high productivity.

Given the potential and needs of these capabilities, in a modern manufacturing environment, it seems reasonable to continue researching and developing of the client applications and monitoring software. A future work is represented by the further implementation of the proposed algorithm in a client and monitoring software implemented on a serve directly connected to the mechatronic line.
The real-time integration of the optimization in a flexible manufacturing system will lead to high performance results in managing the Big Data available in such systems.

\section{Acknowledgements}

The research provided in this work was supported by the Romanian Executive Unit of Funding Higher Education, Research, Development and Innovation (UEFISCDI), project number: PN-IIIP1-1.2-PCCDI-2017-0290, project title: Intelligent and distributed control of 3 complex autonomous systems integrated into emerging technologies for medical-social personal assistance and servicing of precision flexible manufacturing lines.

\section{REFERENCES}

Abidi, M. H., Alkhalefah, H., Mohammed, M. K., Umer, U. \& Qudeiri, J. E. A. (2020). Optimal Scheduling of Flexible Manufacturing System Using Improved Lion-Based Hybrid Machine Learning Approach, IEEE Access, 8, 96088-96114. DOI: 10.1109/ACCESS.2020.2997663

Basir, R., Qaisar, S., Ali, M., Aldwairi, M., Ashraf, M. I., Mahmood, A. \& Gidlund, M. (2019). Fog Computing Enabling Industrial Internet of Things: State-of-the-Art and Research Challenges, Sensors, 19(21), 4807. DOI: 10.3390/s19214807

Bouabid, H., Dhouib, K. \& Gharbi, A. (2012). Zero Inventory/Production Control Policy for Manufacturing Systems Subject to Quality Deterioration. In Proceedings of the 3rd 2012 International Conference on Industrial Engineering and Operations Management, Istanbul, Turkey (pp. 1840-1847).

Boysen, N. (2010). Truck scheduling at zero-inventory cross docking terminals, Computers \& Operations Research, 37(1), 32-41.

Brusaferri, A., Ballarino, A. \& Carpanzano, E. (2011). Reconfigurable Knowledge-based Control Solutions, Studies in Informatics and Control, 20(1), 31-42. DOI: $10.24846 / v 20 i 1 y 201103$

Castano, F., Strzelczak, S., Villalonga, A., Haber, G., Rodolfo, E. \& Kossakowska, J. (2019). Sensor Reliability in Cyber-Physical Systems Using Internetof-Things Data: A Review and Case Study, Remote Sensing, 11(19), 2252. DOI: 10.3390/rs11192252

Ceptureanu, S. I., Ceptureanu, E. G., Popescu, D. I. \& Orzan, O. A. (2020). Eco-innovation Capability and Sustainability Driven Innovation Practices in Romanian SMEs, Sustainability, 12(17), 7106. DOI: 10.3390/su12177106

Cogliati, D. Falchetto, M., Pau, D., Roveri, M. \& Viscardi, G. (2018). Intelligent Cyber-Physical Systems for Industry 4.0. In 2018 First International Conference on Artificial Intelligence for Industries (AI4I), (pp. 19-22). DOI: 10.1109/AI4I.2018.8665681

Dragomir, F., Mincă, E., Dragomir, O. \& Filipescu, A. (2019). Modelling and Control of Mechatronics Lines Served by Complex Autonomous Systems, Sensors, 19(15), 3266. DOI: 10.3390/s19153266

Duca, O., Gurgu, V., Minca, E., Filipescu, A., Dragomir, F. \& Dragomir, O. (2019). Optimal control of the complete assembly/disassembly cycle for a mechatronics line prototype. In 2019 23rd International Conference on System Theory, Control and Computing (ICSTCC), Sinaia (pp. 620-625).

Esheiba, L., Elgammal, A. \& El-Sharkawi, M. (2019). Recommendation Framework for on-Demand Smart Product Customization. In Proceedings of the 21st International Conference on Enterprise Information Systems (ICEIS 2019), Heraklion, Crete, Greece (pp. 177-187).

Filipescu, A., Mincă, E., Filipescu, A. \& Coandă, H.-G. (2020). Manufacturing Technology on a Mechatronics Line Assisted by Autonomous Robotic, Actuators, 9(4), 127. DOI: 10.3390/act9040127

Geismar, H., Dawande, M. \& Sriskandarajah, C. (2011). Pool-Point Distribution of Zero-Inventory Products, Production and Operations Management, 20(5), 737753. DOI: $10.1111 /$ j.1937-5956.2010.01190x 
Gharge, A., Kara, M., Moradlou, H. \& Goswami, M. (2020). The impact of Industry 4.0 implementation on supply chains, Journal of Manufacturing Technology Management, 31(4), 669-686.

Herceg, I., Kuc, V., Mijuskovic, V. \& Herceg, T. (2020). Challenges and Driving Forces for Industry 4.0 Implementation, Sustainability, 12(10), 4208. DOI: $10.3390 /$ su 12104208

Hernandez-Martinez, E. G. \& Puga-Velazquez, E. S. (2013). Modeling Framework for Automated Manufacturing Systems Based on Petri Nets and ISA Standards, Studies in Informatics and Control, 22(2), 163-174. DOI: 10.24846/v22i2y201306

Kagermann, H., Wahlster, W. \& Helbig, J. (2013). Recommendations for implementing the strategic initiative INDUSTRIE 4.0: Securing the future of German manufacturing industry. Final report of the Industrie 4.0 Working Group. acatech -- National Academy of Science and Engineering, München.

Lee, S., Olson, D. \& Trimi, S. (2012). Co-innovation: convergenomics, collaboration, and co-creation for organizational values, Management Decision, 50(5), 817-831.

Luo, H., Yang, X. \& Wang, K. (2019). Synchronized Scheduling of Make to Order Plant and Cross-Docking Warehouse, Computers \& Industrial Engineering, 138, 106108. DOI: 10.1016/j.cie.2019.106108

Metea, S., Çilb, Z., Celika, E. \& Ozceylanc, E. (2019) Supply-driven rebalancing of disassembly lines: A novel mathematical model approach, Journal of Cleaner Production, 213, 1157-1164.

Minca, E. (2014). A New Approach for the Control Optimization of an Assembly/Disassembly Mechatronics Line Served by an Autonomous Robotic System, Studies in Informatics and Control, 21(1), 1322. DOI: $10.24846 / v 23 i 1 y 201402$

Minca, E, Coanda, H.-G., Dragomir, F, Dragomir, O \& Filipescu, A. (2015). Cycle time optimization of a reversible A/DML served by a mobile robotic system. In 19th International Conference on System Theory, Control and Computing (ICSTCC), Cheile Gradistei, Romania (pp. 99-104).

Mourtzis, D., Samothrakis, V., Zogopoulos, V. \& Vlachou, E. (2019). Warehouse Design and Operation using Augmented Reality technology: A Papermaking Industry Case Study, Procedia CIRP, 79, 574-579.

Nagy, G., Illés, B. \& Bányai, A. (2018). Impact of Industry 4.0 on production logistics. In IOP Conference Series Materials Science and Engineering, 448(1), 012013. DOI: 10.1088/1757-899X/448/1/012013

Obermaier, R. \& Donhauser, A. (2012). Zero inventory and firm performance: A management paradigm revisited, International Journal of Production Research, 50(16), 1-13.

Okano, M. (2017). IOT and Industry 4.0: The Industrial New Revolution. In International Conference on Management and Information Systems (ICMIS-17), Bangkok (pp. 75-82).

Özgüven, C., Özbakır, L. \& Yavuz, Y., (2010), Mathematical models for job-shop scheduling problems with routing and process plan flexibility, Applied Mathematical Modelling, 34(6), 1539-1548. DOI: 10.1016/j.apm.2009.09.002

Piccarozzi, M., Aquilani, B. \& Gatti, C. (2018). Industry 4.0 in Management Studies: A Systematic Literature Review, Sustainability, 10(10), 3821. DOI: 10.3390/su10103821

Popescu, D. I., Ceptureanu, S. I., Alexandru, A. \& Ceptureanu, E. G., (2019). Relationships between Knowledge Absorptive Capacity, Innovation Performance and Information Technology. Case study: the Romanian Creative Industries SMEs, Studies in Informatics and Control, 28(4), 463-476. DOI: $10.24846 / v 28 i 4 y 201910$

Poschmann, H., Brüggemann, H. \& Goldmann, D. (2020). Disassembly 4.0: A Review on Using Robotics in Disassembly Tasks as a Way of Automation, Chemie Ingenieur Technik, 92(4), 341-359.

Raposo, D., Rodrigues, A., Sinche, S., \& Sá Silva, J. \& Boavida, F. (2018). Industrial IoT Monitoring: Technologies and Architecture Proposal, Sensors, 18, 3568. DOI: $10.3390 / \mathrm{s} 18103568$

Ren, Y., Meng, L., Zhang, C., Lu, Q. \& Tian, G. (2019). Multi-criterion decision making for disassembly line balancing problem, Procedia CIRP, 80, 542-547.

Rezig, S., Ezzeddine, W., Turki, S. \& Rezg, N. (2020). Mathematical Model for Production Plan Optimization - A Case Study of Discrete Event Systems, Mathematics, 8(6), 955. DOI: https://doi. org/10.3390/math8060955

Rivera-Gómez, H, Montaño-Arango, O., CoronaArmenta, J. R., Garnica-González, J., Ortega-Reyes, A. O. \& Anaya-Fuentes, G. E. (2019). JIT Production Strategy and Maintenance for Quality Deteriorating Systems, Applied Sciences, 9(6), 1180. DOI: 10.3390/ app9061180

Roscoe, S. \& Baker, P. (2013). Supply chain segmentation in the sporting goods industry, International Journal of Logistics Research and Applications, 17(2), 136-155.

Satoglu, S., Ustundag, A., Cevikcan, E. \& Durmusoglu, M. (2017). Lean Production Systems for Industry 4.0, Industry 4.0: Managing the Digital Transformation, 43-59. Springer International Publishing, Switzerland. 
Sousa, R. \& Silveira, G. (2019). The relationship between servitization and product customization strategies, International Journal of Operations \& Production Management, 39(3), 454-474.

Telukdarie, A. Buhulaiga, E., Bag, S., Gupta, S. \& Luo, S. (2018). Industry 4.0 Implementation for Multinationals, Process Safety and Environmental Protection, 118, 316-329.

Tiwong, S., Rauch, E., Soltysova, Z. \& Ramingwong, S. (2019). Industry 4.0 for Managing Logistic Service Providers Lifecycle. In MATEC Web of Conferences, 301(7), 00014. DOI: 10.1051/ matecconf/201930100014

Trabelsi, W., Sauvey, C. \& Sauer, N. (2011). Complexity and Mathematical Model for Flowshop Problem Subject to Different Types of Blocking Constraint, IFAC Proceedings Volumes, 44(1), 81838188. DOI: 10.3182/20110828-6-IT-1002.01887

Vogel, W. (2017). Complexity Management Approach for Resource Planning in Variant-rich Product
Development, Supply Management Research. Advanced Studies in Supply Management, 83-128. Springer Gabler, Wiesbaden.

Westkämper, E. \& Löffler, C. (2016). Implementation of Strategies in the Production System, Strategies of Production, 239-289. Springer-Verlag Berlin Heidelberg.

Westkämper, E., Spath, D., Constantinescu, C., Lentes, J. (Eds.) (2013). Digital Production. Springer, Berlin, Heidelberg.

Zezulka, F., Marcon, P., Bradac, Z., Arm, J., Benesl, T. \& Vesely, I. (2018). Communication Systems for Industry 4.0 and the IIoT, IFAC-PapersOnLine, 51(6), 150-155.

Zhou, J., Luo, J., Lefebvre, D. \& Li, Z. (2020). Modeling and Scheduling Methods for Batch Production Systems Based on Petri Nets and Heuristic Search, IEEE Access, 8, 163458-163471. DOI: 10.1109/ACCESS.2020.3020805 